\title{
Methods of estimating the pooled effect size under meta-analysis: A comparative appraisal
}

\author{
Mona Pathak $^{\mathrm{a}, \mathrm{b}}$, Sada Nand Dwivedi ${ }^{\mathrm{b}, *}$, Bhaskar Thakur ${ }^{\mathrm{a}}$, Sreenivas Vishnubhatla ${ }^{\mathrm{b}}$

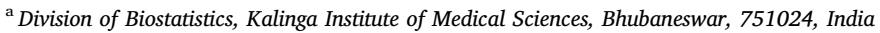 \\ ${ }^{\mathrm{b}}$ Department of Biostatistics, All India Institute of Medical Sciences, New Delhi, 110029, India
}

\section{A R T I C L E I N F O}

\section{Keywords:}

Coverage probability

Fixed effect methods

Precision

Random effects method

Weighted least square method

\begin{abstract}
A B S T R A C T
Introduction: Present study has compared methods of synthesizing the pooled effect estimate under meta-analysis, namely Fixed Effect Method (FEM), Random Effects Method (REM) and a recently proposed Weighted Least Square (WLS) method.

Methods: Three methods of estimating pooled effect estimates under meta-analysis were compared on the basis of coverage probability and width of confidence interval. These methods were compared for seven outcomes with varying heterogeneity and sample size using real data of systematic review comparing neo-adjuvant chemotherapy with adjuvant chemotherapy involving 'hazard ratio' and 'risk ratio' as effect size.

Results: WLS method was found to be superior to FEM having higher coverage probability in case of heterogeneity. Further, WLS with similar coverage probability was found to be superior to REM with more precise confidence interval.

Conclusion: Unrestricted WLS method needs to be preferred unconditionally over fixed effect method and random effects method.
\end{abstract}

\section{Introduction}

Meta-analysis involves statistical approaches to systematically assess the existing evidence, and synthesize them to derive conclusion regarding good clinical/public health practice. The choice of metaanalysis method depends on the extent of heterogeneity (relative excess of between study variance over within study variance). Synthesizing method may affect the pooled effect size which may further alter the conclusion of the study. As a convention, Fixed Effect Method (FEM) is used in the case of homogeneity of the effect sizes while Random Effects Method (REM) is used in the case of heterogeneity. ${ }^{1,2}$ The FEM assumes that all of the studies are measuring the single true effect size which is fixed, and the variation in the observed effect size is just because of sampling fluctuations. ${ }^{2}$ So it considers one source of variation, i.e., within study variance only. However, REM allows variation in the individual study's true effect size, which may occur because of variation in characteristics of participants, intervention, comparison, outcome and design of the study. REM assumes that the effect sizes of the considered studies are a random sample of a large number of such studies (2). It estimates the mean of the distribution of these studies. Because of incorporation of between study variance $\left(\tau^{2}\right)$, REM is used for heterogeneous study level effect sizes. In addition, REM is very sensitive about the method used to calculate $\tau^{2}$, as the pooled effect estimate under REM may vary according the method used for estimating $\tau^{2} .{ }^{3}$ Like REM, another meta-analysis method, unrestricted weighted least square (WLS) method also incorporates the between study heterogeneity. Weighted least squares regression has well defined statistical properties but recently introduced in meta-analysis. ${ }^{4}$ Stanley and Doucouliagos using simulated data demonstrated that unrestricted WLS method performed better than FEM in terms of coverage probability. Further, in case of publication or small sample bias, WLS was superior also to REM with relatively less bias and Mean Square Error (MSE).

The relative performance of these methods have not been examined regarding precision or width of confidence interval despite the fact that it has impact on the statistical significance of the result. Many RCTs, especially in cancer research deals with survival outcomes involving hazard ratio as effect size. The performance of the three methods has not yet been compared for hazard ratio. Also, observed data may be distributed differently from the simulated one as distribution of effect size may not exactly follow theoretical distribution. Accordingly, the present study is aimed to compare the above mentioned three methods of estimating pooled effect size using real data on breast cancer and using two effect sizes as hazard ratio and risk ratio, in terms of coverage probability and precision.

\footnotetext{
* Corresponding author. Department of Biostatistics, All India Institute of Medical Sciences, Ansari Nagar, New Delhi, 110029, India.

E-mail addresses: dwivedi7@hotmail.com,dwivedi7@gmail.com,dwivedi7@aiims.edu, dwivedi@aiims.ac.in (S.N. Dwivedi).
} 


\section{Materials and methods}

The methods under consideration were compared using meta-analysis of data available under a systematic review of randomized controlled trials assessing efficacy of neo-adjuvant chemotherapy in comparison to adjuvant chemotherapy in the management of breast cancer (accepted for publication). ${ }^{5}$ The published articles in English language through comprehensive search of PubMed and Cochrane database with a pre-defined search strategy were considered. Eighteen studies eligible for meta-analysis were identified after screening of titles and abstracts followed by full text review. This systematic review involved overall survival, disease free survival, relapse free survival, loco-regional recurrence, local recurrence, regional recurrence and breast conserving surgery. Hazard ratios (HR) were synthesized for all of the outcomes except breast conserving surgery for which Risk Ratio (RR) was synthesized. The statistical heterogeneity was assessed by $\mathrm{I}^{2}$ statistic. ${ }^{6,7}$ The details of outcome definitions and related results are provided in the manuscript accepted for publication elsewhere. ${ }^{5}$

\subsection{Methods under meta-analysis}

The pooled effect under meta-analysis is weighted average of the study level effect sizes. The only thing which differs in various synthesizing methods is the calculation of weights and how these weights incorporate between study heterogeneity. All the three methods consider different weights for the calculation of pooled effect. Though there are different types of weights, the present study considered only inverse variance weights. ${ }^{8}$ As per objective, the pooled effect estimates were obtained under three methods described below:

\subsection{Fixed effect method}

The fixed effect method ${ }^{2}$ assumes that each study measures a single fixed effect size $(\mu)$ and variation in the effect size (if any) is due to chance alone. Statistically, it assumes that the effect size $\left(\mathrm{y}_{\mathrm{i}}\right)$ follows a normal distribution with mean $(\mu)$ and variance $\left(v_{i}\right)$, i.e., $\mathrm{y}_{i} \sim N\left(\mu, v_{i}\right)$ where $v_{i}=\sigma_{i}^{2}$. The individual study effect size can be presented as $y_{i}=\mu+\varepsilon_{i}$ where $\varepsilon_{i}$ is the sampling error and $\varepsilon_{i} \sim N\left(0, \sigma_{i}^{2}\right)$ for $\mathrm{i}=1,2$ $\ldots \mathrm{k} ; \mathrm{k}$ is the number of studies included in the meta-analysis. The weight $\mathrm{w}_{\mathrm{i}}$ associated with ith study is the inverse of the variance $\left(\sigma_{i}^{2}\right)$ of effect size of the considered outcome, $w_{i}=1 / \sigma_{i}^{2}$ for that study. The pooled effect size $\left(\hat{\mu}_{F}\right)$ considering these weights may be obtained as:

$\hat{\mu}_{F}=\sum y_{i} w_{i} / \sum w_{i}$

Further, the variance of this pooled effect size is the inverse of sum of weights:

$V\left(\hat{\mu}_{F}\right)=1 / \sum w_{i}$

The pooled effect estimate under FEM is also used to calculate Q statistic, to test absence of the heterogeneity in the meta-analysis. The $\mathrm{Q}$ statistic is defined as the weighted sum of square of deviation of individual effect size from pooled effect size computed by FEM. ${ }^{9}$ Further, weights used in calculating pooled effect size are also retained.

$Q=\sum w_{i}\left(y_{i}-\hat{\mu}_{F}\right)^{2}$

\subsection{Random effects method}

Unlike FEM, REM ${ }^{10,11}$ assumes that study level effect size is a random sample out of the infinite similar studies. REM considers that study level effect size may vary systematically because of change in its population, intervention, comparator, outcome definition and used design. In other words, the deviation $\left(\theta_{i}\right)$ between individual study effect size $\left(y_{i}\right)$ and the true effect in the population $(\mu)$ is more than that due to sampling variation $\left(\varepsilon_{i}\right)$ alone. The individual study effect size can be modeled as:

$y_{i}=\mu+\theta_{i}+\varepsilon_{i}$

Further, $\theta_{i}$ depends on between study variance $\left(\tau^{2}\right)$ while $\varepsilon_{i}$ depends on the within study variance $\left(\sigma_{i}^{2}\right)$ alone. As REM considers random sample of the effect sizes, the mean of the deviation in effect size is zero. REM assumes that $\theta_{i}$ is distributed normally with mean zero and variance $\left(\tau^{2}\right)$ as $\theta_{i} \sim N\left(0, \tau^{2}\right)$ and sampling error is also distributed normally as $\varepsilon_{i} \sim N\left(0, \sigma_{i}^{2}\right)$. Since REM considers two sources of variation, between study variance as well as within study variance, the weight $\left(w_{i}^{\prime}\right)$ associated with individual study is inverse of this total variation:

$w_{i}^{\prime}=1 /\left(\sigma_{i}^{2}+\tau^{2}\right)$

The pooled effect estimate of $y_{i}$ may be given as below:

$\hat{\mu}_{R}=\sum y_{i} w_{i}^{\prime} / \sum w_{i}^{\prime}$

where $y_{i}$ is distributed as. $y_{i} \sim N\left(\mu, \sigma_{i}^{2}+\tau^{2}\right)$

Further, the variance of this estimated effect size may be represented as:

$V\left(\hat{\mu}_{R}\right)=1 / \sum w_{i}^{\prime}$

Since REM considers between study variance as well, it may result into larger variance of effect size which may further lead to wider confidence interval of pooled effect estimate in comparison to pooled effect estimate under FEM. However, in case of no heterogeneity, i.e., $\tau^{2}=0$ the pooled estimate as well as its $95 \% \mathrm{CI}$ will coincide with that under FEM.

The pooled effect estimates under REM depends on the estimated value of between study variance. The between study variance can be estimated by various available methods. In our another exploration, it was found that DerSimonian \& Laird method and Hunter \& Schmidt ${ }^{12}$ method are best among all available methods of estimating between study variance under random effects method. As DerSimonian and Liard Method is available in most of the statistical software, present study preferred to use it for estimating $\tau^{2}$ under REM.

\subsection{Weighted least square method}

The third method under consideration, unrestricted weighted least square method, was recognized by Stanley and Doucouliagos ${ }^{4}$ for synthesizing the effect sizes. The WLS method assumes the same model as discussed under FEM, i.e., $y_{i}=\mu+\varepsilon_{i}$ where $y_{i} \sim N\left(\mu, v_{i}\right)$. Also, it assumes that the variance of the effect estimate $\left(\mathrm{v}_{\mathrm{i}}\right)$ can be estimated up to some unknown multiplicative coefficient $(\phi)$. So, the variance, $v_{i}=\varphi \sigma_{i}^{2}$. Thus weight of individual study effect size $\left(\mathrm{w}_{\mathrm{i}}{ }^{*}\right)$ becomes:

$w_{i}^{*}=\frac{1}{\varphi \sigma_{i}^{2}}=\frac{w_{i}}{\varphi}$

where $\mathrm{w}_{\mathrm{i}}$ are weights under FEM.

The point estimate under the WLS method will be same as under FEM because the multiplicative constant $\phi$ will get cancelled out while calculating the weighted average:

$\hat{\mu}_{\mathrm{W}}=\frac{\sum \mathrm{y}_{\mathrm{i}} \mathrm{w}^{*}{ }_{\mathrm{i}}}{\sum \mathrm{w}_{\mathrm{i}}^{*}}=\hat{\mu}_{\mathrm{F}} \forall(\varphi \neq 0)$

But the variance of the pooled effect estimate under WLS will differ from that of FEM, as it is $\phi$ times of that under FEM. The variance can be described as:

$\mathrm{V}\left(\hat{\mu}_{\mathrm{W}}\right)=\frac{1}{\sum \mathrm{w}_{\mathrm{i}}^{*}}=\frac{\varphi}{\sum \mathrm{w}_{\mathrm{i}}}=\varphi \mathrm{V}\left(\hat{\mu}_{\mathrm{F}}\right)$

Under WLS method, the width of confidence interval will depend on the estimated value of $\phi$ and it will obviously rely on the extent of heterogeneity. The estimate of $\phi$ can be obtained from the MSE of the simple meta-regression of the standardized effect size with precision(4) 
which is also equal to the one of the heterogeneity measure $\mathrm{H}^{2}$.

$\varphi=\mathrm{H}^{2}=\frac{\mathrm{Q}}{\mathrm{k}-1}$

For considered number of studies ( $\mathrm{k}), \mathrm{H}^{2}$ can also be calculated from $\mathrm{Q}$ (equation (1)).

Since $\mathrm{I}^{2}$ statistic is widely calculated in most of the software, $\mathrm{H}^{2}$ can also be calculated from $\mathrm{I}^{2}$ as:

$\mathrm{H}^{2}=\frac{1}{\left(1-\mathrm{I}^{2}\right)}$

As $\mathrm{H}^{2}=1$ in the case of no heterogeneity but increases with the increase in the heterogeneity, so $\phi \geq 1$. This implies that CIs under WLS are always either equal or wider than that of under FEM. Further, wider CIs result into higher p-value under WLS in comparison to FEM.

\subsection{Comparison of meta-analysis methods}

An ideal method should have minimum bias, low MSE, higher coverage probability ${ }^{4}$ but with narrow confidence interval. Since, instead of simulation data, we dealt with observed data, bias and mean square error calculation were not possible. Accordingly, the performance of FEM, REM and WLS methods was compared using coverage probability and precision for each outcome.

Coverage Probability and Precision: It is the proportion of times the 95\% confidence interval contains the population parameter. ${ }^{13,14}$ The coverage probability of interval estimates synthesized by each of the three methods was calculated using 10000 bootstrap samples of studies under meta-analysis. The pooled effect estimate by each of the three methods was calculated for each of these 10000 samples which obviously ensured same heterogeneity of samples under each method. Further, the proportion of these pooled effect estimates under each of the bootstrap sample lying within the $95 \%$ CI of pooled effect estimate of original set of studies under meta-analysis by respective synthesis method (FEM, REM or WLS) was considered as coverage probability. Along with coverage probability, the width of confidence interval and standard error also matters, so it was also taken into consideration while comparing the considered methods.

Software Used: The analysis was carried out using Stata 13.1, StataCorp, Texas. Stata programs (version 13.1) for WLS meta-analysis with effect size and its standard error as well as for risk ratio with cell parameters were written (appendix).

\section{Results}

Present study compared all the methods, i.e., FEM, REMs and WLS methods for estimation of pooled effect size for various outcomes involving variable heterogeneity and sample size [Table 1]. In case of no heterogeneity, e.g., overall survival, loco-regional recurrence and local recurrence, the point as well as interval estimates of hazard ratio under three methods coincided [Table 1]. With increasing heterogeneity, disparity among precision of various methods also increased.
Specifically, for BCS involving highest heterogeneity, risk ratio $(95 \%$ CI) under fixed effect method, random effects method and weighted least square method are 1.12 (1.07-1.16), 1.21 (1.04-1.41) and 1.12 $(0.98-1.27)$ respectively. Fixed effect method has most precise confidence interval followed by weighted least square method. Regardless of any of the three methods, the width of confidence interval relied on the number of studies under meta-analysis and also on heterogeneity. Further, it decreased with increased number of studies. On the other hand, it increased with increased heterogeneity [Table 1 \& Fig. 1].

In the presence of heterogeneity, FEM performed very poorly in terms of coverage probability as the coverage probability was found to be only 0.5464 for breast conserving surgery, which ideally should have been around 0.95 . In other words, 95\% confidence interval of pooled effect estimate of BCS under FEM actually contains actual effect size only $55 \%$ of the times instead of claiming $95 \%$ of the times. In summary, among all the methods, FEM estimates had most precise confidence interval of pooled effect but with poor coverage probability. It may be mainly due to non-consideration of between study variability. In contrary, the coverage probability of relative risk by WLS method for BCS was 0.9625 . Further, with similar coverage probability and heterogeneity, more precise confidence interval was observed under WLS in comparison to REM. Since estimation of pooled effect estimate and its $95 \%$ confidence interval by each of the three methods was done for same set of 10000 bootstrap samples, heterogeneity may differ from sample to sample but it was same under each of three methods. The standard error under WLS method was relatively less in comparison to that under REM for all bootstrap samples under all considered outcomes. More than $95 \%$ of the times, REM had wider or similar confidence interval in comparison to WLS method [Table 2]. This analysis suggests that the WLS method produced more consistent estimate of pooled effect size in comparison to REM. Also, coverage probabilities under WLS method were superior to that under REM except disease free survival. For disease free survival also WLS method had similar or narrower $95 \%$ CI $(94.5 \%$ of the times) in comparison to REM Pooled effect estimate of disease free survival which was based on only six RCTs. Among these six RCTs, only one RCT was cause of heterogeneity. Multiple times inclusion of that particular RCT in bootstrap sample may be the reason of lowering coverage probability.

\section{Discussion}

In our study on observed data, we also observed, poor coverage probability under FEM in case of heterogeneity as reported earlier. ${ }^{4}$ Further, based on analytical results on simulated data, they suggested to use WLS especially in case of publication bias or small number of studies under meta-analysis. However, keeping in view of results on observed data in present study; unrestricted WLS method needs to be preferred unconditionally. Further, as REM and WLS have provided different conclusions in case of breast conserving surgery involving very high heterogeneity, their clinical interpretation also needs to be considered while choosing the method. For such a high heterogeneity, the

Table 1

Pooled effect estimate for outcomes under fixed effect method, random effects method and unrestricted weighted least square method.

\begin{tabular}{|c|c|c|c|c|c|}
\hline \multirow[t]{2}{*}{ Outcome } & \multirow[t]{2}{*}{ No of RCTs } & \multirow[t]{2}{*}{$\mathrm{I}^{2}$ Statistic (\%) } & \multicolumn{3}{|c|}{ Pooled Effect Size (95\% Confidence Interval) } \\
\hline & & & Fixed Effect Method & Random Effects Method & Weighted Least Square Method \\
\hline Overall Survival & 15 & 0.00 & $0.98(0.89-1.08)$ & $0.98(0.89-1.08)$ & $0.98(0.89-1.08)$ \\
\hline Disease Free Survival & 6 & 26.30 & $0.98(0.87-1.09)$ & $0.99(0.83-1.19)$ & $0.98(0.86-1.11)$ \\
\hline Relapse Free Survival & 14 & 47.20 & $1.04(0.94-1.14)$ & $1.01(0.86-1.18)$ & $1.04(0.91-1.18)$ \\
\hline Loco-Regional Recurrence & 15 & 0.00 & $1.23(1.06-1.43)$ & $1.23(1.06-1.43)$ & $1.23(1.06-1.43)$ \\
\hline Local Recurrence & 10 & 0.00 & $1.31(1.11-1.56)$ & $1.31(1.11-1.56)$ & $1.31(1.11-1.56)$ \\
\hline Distant Metastasis & 13 & 43.50 & $0.98(0.88-1.09)$ & $0.97(0.82-1.16)$ & $0.98(0.85-1.13)$ \\
\hline Breast Conserving Surgery ${ }^{\mathrm{a}}$ & 9 & 90.10 & $1.12(1.07-1.16)$ & $1.21(1.04-1.41)$ & $1.12(0.98-1.27)$ \\
\hline
\end{tabular}

${ }^{a}$ Risk Ratio was considered for breast conserving surgery, hazards ratio for other outcomes. RCT-Randomized controlled trials. 


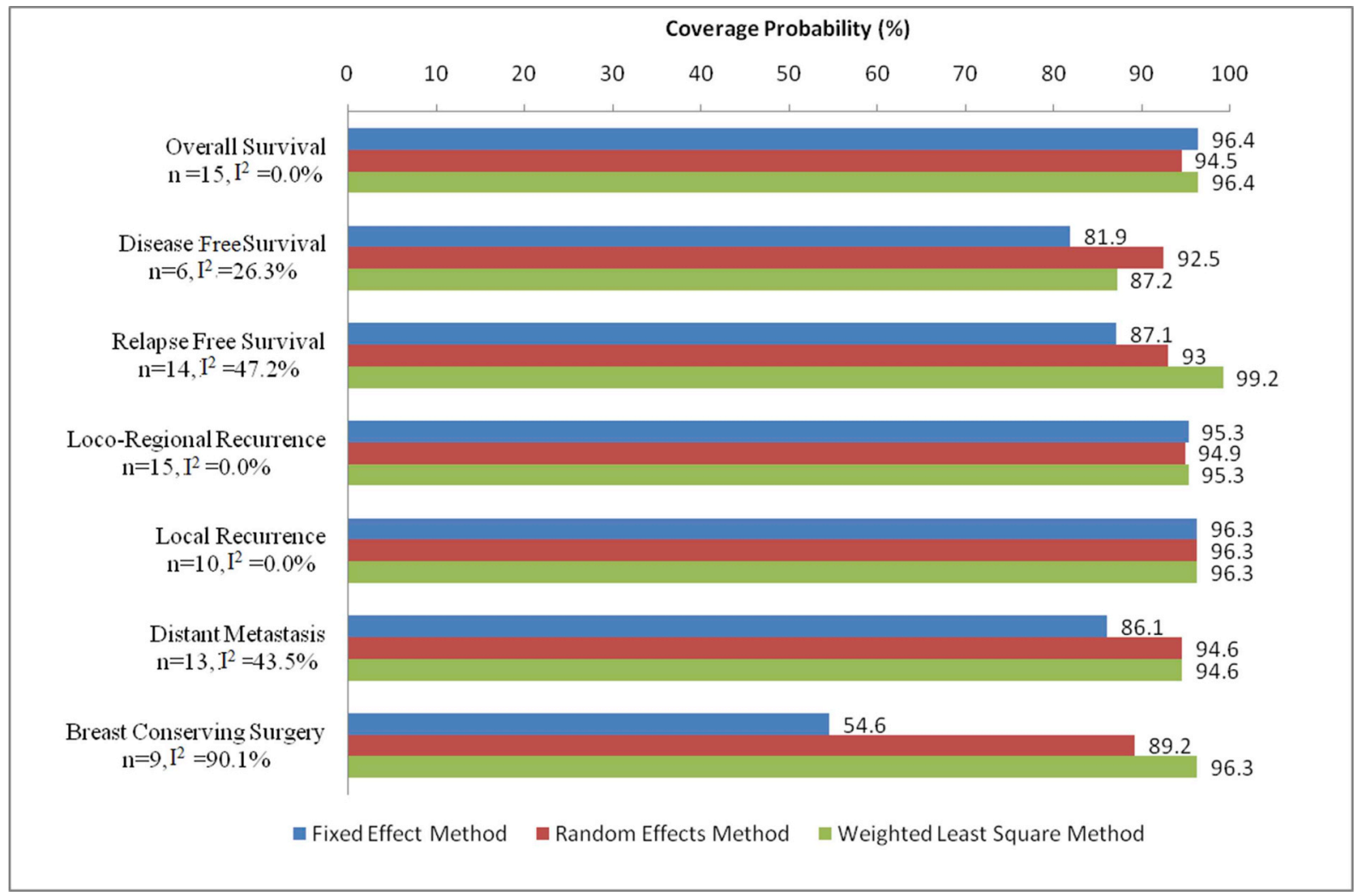

Fig. 1. Coverage Probability (\%) under fixed effect method (FEM), random effects method (REM) and unrestricted weighted least square method (WLS) for the considered outcomes.

Table 2

Comparison of (frequency of shorted, wider and equal) width of $95 \%$ confidence interval for 10000 bootstrap samples of study level effect sizes for random effects method and unrestricted weighted least square method.

\begin{tabular}{|c|c|c|c|}
\hline & $\begin{array}{l}\text { REM }<\text { WLS N } \\
(\%)\end{array}$ & $\begin{array}{l}\text { REM > WLS N } \\
(\%)\end{array}$ & $\begin{array}{l}\text { REM }=\text { WLS N } \\
(\%)\end{array}$ \\
\hline Overall Survival & $1(0.01)$ & $2357(23.57)$ & $7642(76.42)$ \\
\hline Disease free Survival & $550(5.50)$ & $5370(53.70)$ & $4080(40.80)$ \\
\hline Relapse Free Survival & $351(3.51)$ & 9092(90.92) & $557(5.57)$ \\
\hline $\begin{array}{l}\text { Loco-Regional } \\
\text { Recurrence }\end{array}$ & $23(0.23)$ & $3173(31.73)$ & $6805(68.05)$ \\
\hline Local Recurrence & $29(0.29)$ & $3153(31.53)$ & $6818(68.18)$ \\
\hline Distant Metastasis & $153(1.53)$ & 9352(93.52) & 495(4.95) \\
\hline $\begin{array}{l}\text { Breast Conserving } \\
\text { Surgery }\end{array}$ & $1(0.01)$ & 9589(95.89) & $410(4.10)$ \\
\hline
\end{tabular}

REM- Random effects method WLS- Weighted least square method.

cause of heterogeneity needs to be investigated and sensitivity or subgroup analysis should be conducted.

Incapability of fixed effect method to provide reliable estimate in case of heterogeneity is natural as it was designed for homogeneous studies only. But, superiority of WLS over REM needs to be focused. To be more specific, similar point as well as interval estimates were observed under three methods in case of no heterogeneity. But, in case of heterogeneity, WLS revealed higher coverage probability as compared to FEM. Further, although similar coverage probability under both, WLS estimate had highly precise confidence interval in comparison to REM. Also, WLS may be superior to REM, because in contrary to REM, under varying methods to calculate between study variance, it remains same. ${ }^{3}$ For very few number of heterogeneous studies $(<=5)$, REM as well as WLS did not provided desired coverage probability. Further, as we know that the random effects method (REM) using DerSimonian Laird method produces an unreliable estimate for a small number of studies (especially for studies $\leq 5$ ) in a meta-analysis, there is need to extend this study of comparing three methods involving varying methods in calculating between study variance under REM.

\section{Funding}

The present research work was not funded by any funding agency.

\section{Conflicts of interest}

Authors have declared that they have no conflict of interest.

\section{Author's contribution}

All authors conceived \& designed the study. MP did statistical analysis. All authors interpreted the results. The final manuscript of the research work was prepared by MP. All the authors read and provided their inputs to finalize the manuscript.

\section{Author's information}

Currently, Dr. Mona Pathak is Assistant Professor in Kalinga Institute of Medical Sciences, Bhubaneswar, India. She was Ph.D. student under the supervision of Prof. SN Dwivedi at the time of this research work. Dr. SN Dwivedi and Dr. V Sreenivas are professor in All India Institute of Medical Sciences (AIIMS), New Delhi, India. Dr. Bhaskar Thakur is Assistant Professor in Kalinga Institute of Medical Sciences, Bhubaneswar, India.

\section{Acknowledgements}

The authors thank the reviewers for their valuable comments which helped in strengthening the article. The authors thank All India Institute 
of Medical sciences (AIIMS), New Delhi, to make available the computer laboratory facility, library, online accessibility of articles and other resources.

\section{Abbreviations}

FEM

REM

WLS

HR

$\mathrm{RR}$

BCS
Fixed Effect Method

Random Effects Method

weighted least square

Hazard Ratio

Risk Ratio

Breast Conserving Surgery

Appendix A. Supplementary data

Supplementary data to this article can be found online at https://doi.org/10.1016/j.cegh.2019.05.004. 


\section{Appendix}

A.1 Stata program for calculating pooled effect size using weighted least square method

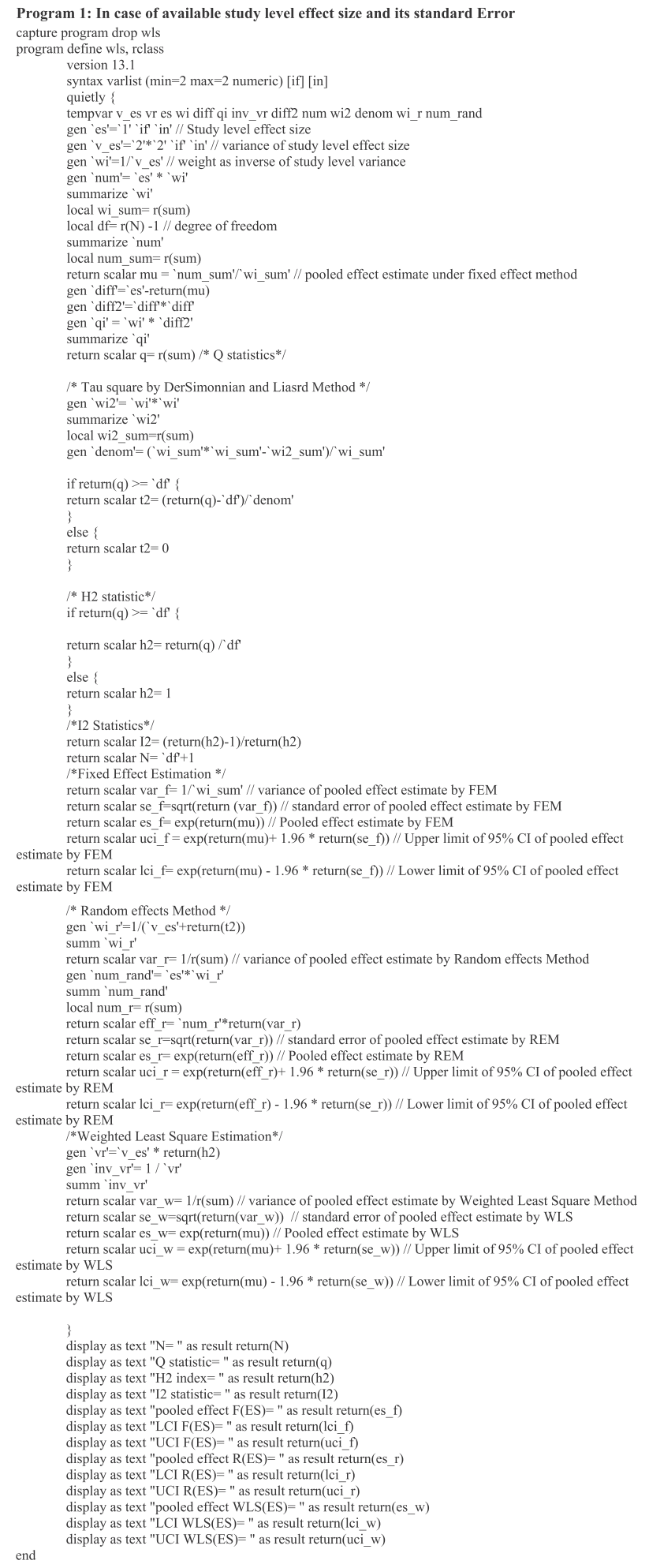




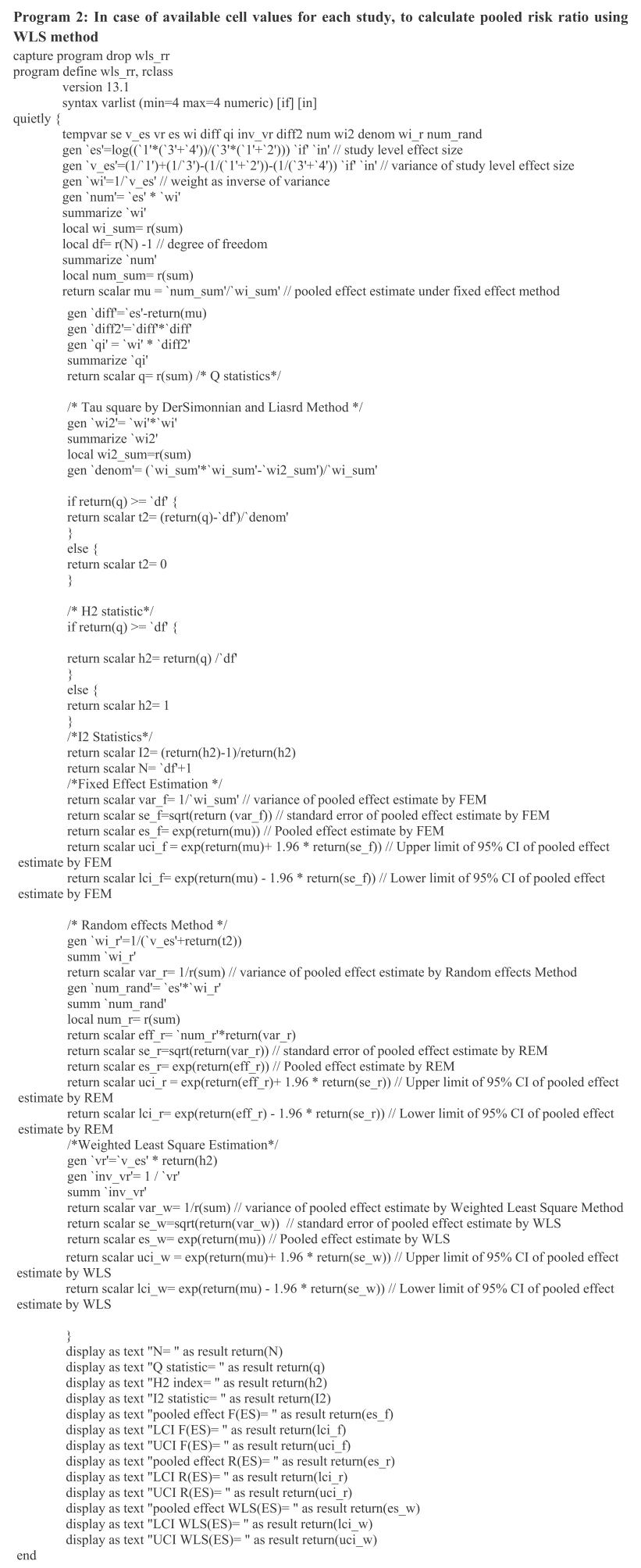

\section{References}

1. Tufanaru C, Munn Z, Stephenson M, Aromataris E. Fixed or random effects metaanalysis? Common methodological issues in systematic reviews of effectiveness. Int $J$ Evid Based Healthc. 2015 Sep;13(3):196-207.

2. Borenstein M, ed. Introduction to Meta-Analysis. Chichester, U.K: John Wiley \& Sons; 2009:421.

3. Veroniki AA, Jackson D, Viechtbauer W, et al. Methods to estimate the between-study variance and its uncertainty in meta-analysis. Res Synth Methods. 2016
Mar;7(1):55-79.

4. Stanley TD, Doucouliagos H. Neither fixed nor random: weighted least squares metaanalysis. Stat Med. 2015 Jun 15;34(13):2116-2127.

5. Pathak M, Deo SVS, Dwivedi SN, et al. Role of neoadjuvant chemotherapy in breast cancer patients: systematic review and meta-analysis. Indian J Med Pediatr Oncol Print [Internet]. 2019 Jan. [cited 2018 Jul 8]; Available from:. http://www.ijmpo.org/ aheadofprint.asp.

6. Higgins JP, Thompson S. Quantifying heterogeneity in a meta-analysis. Stat Med. 2002;21:1539-1551.

7. Pathak M, Dwivedi SN, Deo S, Vishnubhatla S, Thakur B. Which is the preferred 
measure of heterogeneity in meta-analysis and why? A revisit. Biostat Biom Open Acc J. 2017 Mar 27;1(1):55555

8. Schwarzer G, Carpenter JR, Rücker G. Fixed effect and random effects meta-analysis. In: Schwarzer G, Carpenter JR, Rücker G, eds. Meta-Analysis with $R$ [Internet]. Cham: Springer International Publishing; 2015:21-53 [cited 2018 Dec 25]. https://doi.org/ 10.1007/978-3-319-21416-0_2. (Use R!). Available from:.

9. Huedo-Medina TB, Sánchez-Meca J, Marín-Martínez F, Botella J. Assessing heterogeneity in meta-analysis: Q statistic or $\mathrm{I}^{2}$ index? Psychol Methods. 2006;11(2):193-206.

10. DerSimonian R, Laird N. Meta-analysis in clinical trials. Contr Clin Trials. 1986 Sep
1;7(3):177-188

11. DerSimonian R, Kacker R. Random-effects model for meta-analysis of clinical trials: an update. Contemp Clin Trials. 2007 Feb;28(2):105-114.

12. Hunter JE, Schmidt FL. Methods of Meta-Analysis: Correcting Error and Bias in Research Findings. second ed. Thousand Oaks, Calif: Sage; 2004582 pp.

13. Agresti A, Coull BA. Approximate is better than "exact" for interval estimation of binomial proportions. Am Statistician. 1998;52(2):119-126.

14. Coverage probability of confidence intervals. A Simulation Approach - the DO Loop [Internet]. . [cited $2019 \mathrm{Feb} 4$ ]. Available from:. https://blogs.sas.com/content/iml/ 2016/09/08/coverage-probability-confidence-intervals.html. 